\title{
Contour, iDHD, and ICHD Residual Stress Measurements on a T-Section Plate
}

\author{
X. Ficquet ${ }^{1 a *}$, D. Douglas ${ }^{1}$, K. Serasli ${ }^{1}$, F. Bridier ${ }^{2}$ \\ ${ }^{1}$ VEQTER Ltd, 8 Unicorn Business Park, Whitby Road, Bristol BS4 4EX, UK \\ 2 DCNS Research, Technocampus Ocean, 44340 BOUGUENAIS, France \\ axavier.ficquet@veqter.co.uk
}

\section{Keywords: Residual Stress, Contour, iDHD, T-Section, Fillet Weld}

\begin{abstract}
This paper presents a review of work carried out to ascertain the residual stresses present within a T-plate section. The contour method, the incremental deep hole drilling (iDHD) and the incremental centre hole drilling (ICHD) are coupled to determined longitudinal and transverse components of residual stress in the weld tope of the fillet weld in the as-welded condition. This paper presents first the measurement using the iDHD method and the measurement obtained using the contour method is then presented. The accuracy and resolution of the contour method results are directly linked to the quality of the electro- discharge machining (EDM) cut made. The challenges incurs by cutting complex geometry like this T-plate section are identified and their influence on residual stresses calculated by the contour method is quantified.
\end{abstract}

\section{Introduction}

Welding residual stresses play an important role when assessing the structural integrity of welded components. The ability to determine residual stresses at critical locations in components as well as throughout the component for structural integrity assessments is desirable. Current measurement techniques to measure the trough thickness residual stress includes the Incremental Deep Hole Drilling (DHD), Neutron Diffraction (ND) and the Synchrotron Xray (HEXRD). The Contour technique, more recent, is capable of measuring residual stresses in a single direction, in a twodimensional plane through a specimen. A T-plate component is particularly challenging for the contour measurement due to the different change in section.In this paper, the residual stress of the T-section fillet weld has been determined using the iDHD, the contour and the Incremental Center-Hole Drilling (ICHD) techniques. A description of the t-section is first provided. A brief description of the iDHD technique is provided with a more details description of the contour technique. The contour measurement is described stage by stage, and highlight the problem when a wire breaks during the wire-EDM cutting process.

\section{Component description and measurement locations}

The component characterised in the present work comprises a main plate with a web stiffener plate fillet welded on the top surface of the main plate. A smaller flange plate is also fillet welded on the top of the web plate. All these components are made of high strength steel. The web plate was welded on both the main and flange plates using an automatic arc welding processes with a simultaneous welding on both sides of the web plate.

The iDHD measurement was carried out first, located at $207 \mathrm{~mm}$ longitudinally away from the front of the specimen at weld toe 1 and carried out to a depth of 0.44 to from the weld toe surface through the hull plate, where to is the thickness of the hull plate, see Fig. 1. Then the contour measurement was performed

$70 \mathrm{~mm}$ longitudinally away from the iDHD measurement towards the front end of the specimen. The contour cut was made at $90^{\circ}$ to the fillet welds in order to obtain a map of the longitudinal residual stresses. 
Finally, a total of three ICHD measurements were carried out on the Contour cut surfaces after completion of the Contour measurement, see Fig. $2 \mathrm{~b}$. ICHD1 was located at 0.1 t 0 below the weld toe 2 , ICHD2 was located at the same depth below weld toe1. ICHD3 was located at $0.18 \mathrm{t}_{0}$ below weld toe 1 on the opposite contour cut surface to ICHD 2.

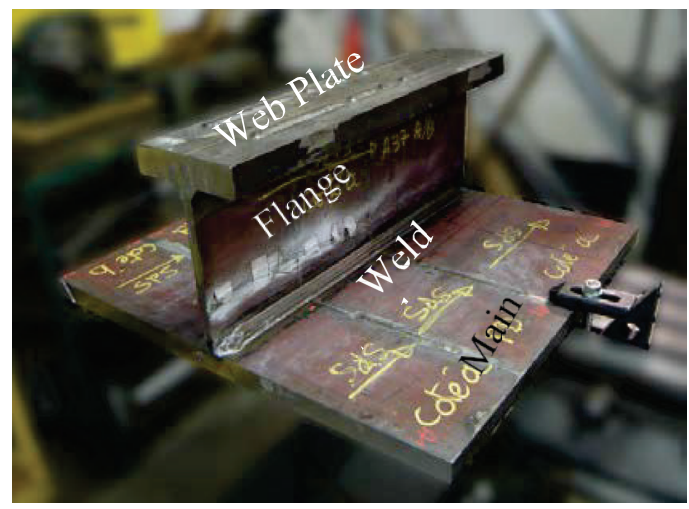

(a)

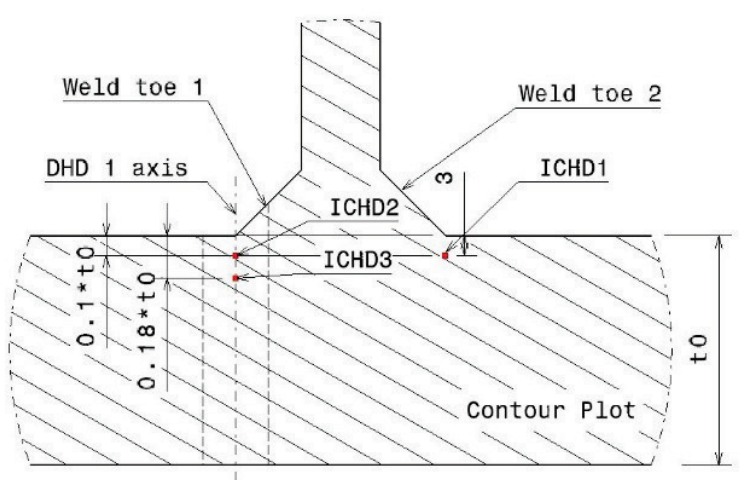

Fig. 1: (a) Distorted photograph of the specimen, (b) Drawing showing the location of the ICHD measurements on the Contour measurement surface.

\section{Residual Stress Measurement description}

In order to define the full residual stress distribution in the joints two techniques were employed. The well-established technique iDHD was combined with the contour method, a more recent technique. The iDHD technique can provide bi-axial residual stress through the thickness of the joint. This technique can measure steep gradient through the thickness, however the results are only provided along the measurement line. The contour method has the advantage of measuring the full 2D uni-axial residual stress normal to a cut. Therefore providing the location of the maximum and minimum residual stress peaks. The iDHD technique has been covered in numerous papers, therefore only a brief description of the technique will be provided. The contour measurement will be developed in more details.

The iDHD measurement. The DHD technique is a semi-invasive, mechanical strain relief technique (i.e. the strain of the component is measured during stress relief from the removal of a small amount of material). The analysis of measured distortion was developed by Leggatt et al [1] and later extensively refined, [2]. The iDHD technique can be applied in two ways depending on the residual stresses assumed to be present within the component. The standard technique (DHD) involves trepanning through the component in a single stage and then measuring the final diameter of the deformed reference hole. However, if the component contains, for example, high magnitude, tri-axial residual stresses then during the trepanning process the material will undergo plastic relaxation and the deformed shape of the reference hole will not be representative of the original residual stress field and so provide inaccurate results. Therefore if it is thought that plasticity will occur during the DHD process then the modified technique, called Incremental-DHD or iDHD, will be used in which the trepanning process is carried out in multiple stages and the diameter of the reference hole is measured after each trepanning stage. With the modified technique plasticity is no longer an issue.

In order to estimate where plastic relaxation may occur, finite element (FE) analysis predictions of the residual stresses generated in similarly designed and manufactured components and/or previous measurements are reviewed. It was decided that for this iDHD measurement, a single incremental EDM step would be performed at approximately $0.8 \mathrm{t}_{0}$ deep from the fillet weld toe. With iDHD, a single, biaxial measurement point is produced per EDM increment, usually within a distance of $+2 \mathrm{~mm}$ from the EDM increment depth. Unlike the standard DHD technique where 
measurement points at specific depths can be prescribed, the iDHD technique cannot be so exact. The incremental depth of the EDM processcan be prescribed, but the location of the measured point depends upon the magnitude of residual stresses present and the level of plastic relaxation.

The contour measurement. The contour residual stress measurement technique is a destructive mechanical strain relief technique. The technique is based on a variation of Bueckner's elastic principle of superposition [3]. The technique provides a full uniaxial map of the residual stress perpendicular to a cut plane through the specimen. The contour method can be divided into 5 stages.

Stage 1: Sacrificial bushes, highlighted in blue in Fig. 2a, are attached to the specimen with conductive adhesive in line with the plane of measurement. A small pilot hole is then drilled through the bushes and specimen along the measurement plane, at $90^{\circ}$ to the cutting direction and away from the zone of most interest to permit "self-constraint" during the cut. The sacrificial material attached to the specimen creates a constant cut area throughout the specimen and aids to cutting accuracy and prevent wire breakages $[3,4]$.

Stage 2: The specimen is clamped into a wire-EDM (electric-discharge machining) machine, and cut into two sections in a single pass with a $250 \mu \mathrm{m}$ diameter brass wire on "skim cut" settings. The cut is initiated from the pilot hole which keeps the specimen constrained. This is of critical importance during the wire-EDM cutting process in order to minimise plastic deformations and bulge error [4]. The cut creates two stress-free surfaces and hence relaxes the out-of-plane residual stresses.

The complex geometry of the specimen made the fitting and attachment of the sacrificial material difficult. The EDM wire may have vibrated due to the various transitions from cutting through the bushes to cutting through the specimen. Cutting artefacts were therefore left apparent on the cut surface in line with these various transitions, shown in Fig. $2 b$.

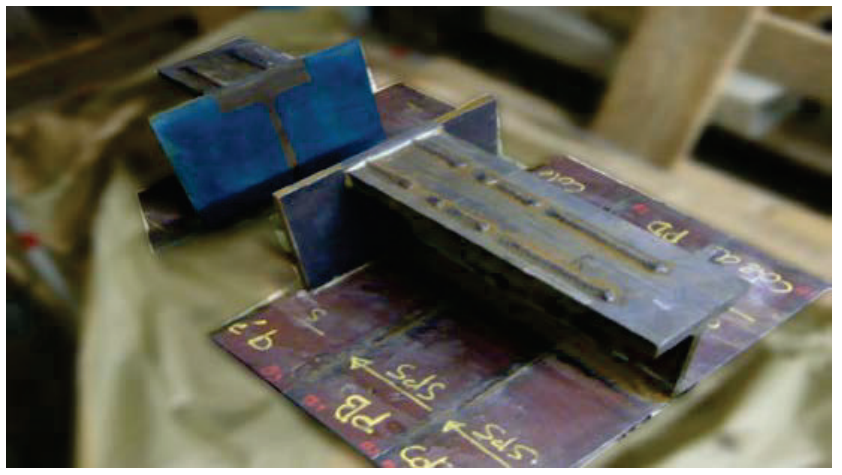

(a)

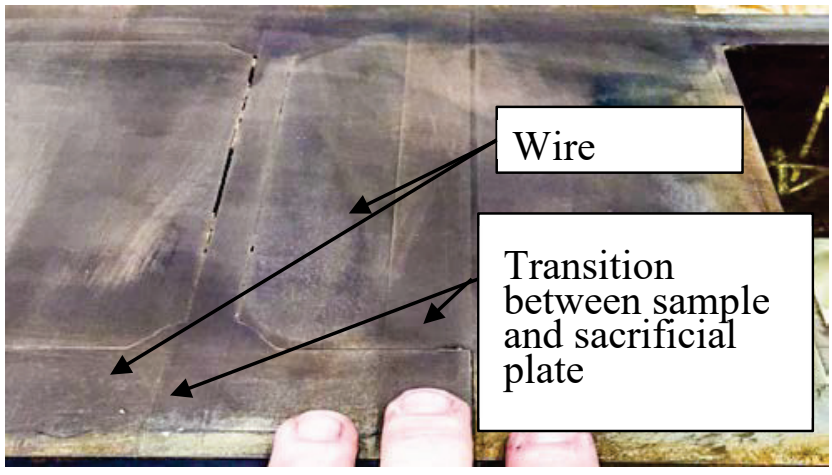

(b)

Fig. 2: (a) Cross section showing sacrificial materials, (b) The wire breaks and artefacts on the cut surface

Stage 3: The two newly cut surfaces are measured with a coordinate measuring machine (CMM) to record their out-of-plane deformations resulting from the relaxation of the out-of-plane residual stresses. Therefore the longitudinal displacements of both contour cut surfaces due to longitudinal residual stress relaxation were measured using CMM with a node spacing of about $0.2 \mathrm{~mm}$ in the weld regions and about

$0.5 \mathrm{~mm}$ elsewhere. The $6 \mathrm{~mm}$ diameter, spherical probe tip of the CMM provides an uncertainty of about $\pm 4 \mu \mathrm{m}$.

A wire break near the weld toe during the EDM cutting meant that an important region of data was corrupted. As the residual stress profile was considered to be symmetrical about the stiffener, the 
wire break zone was repaired with mirrored data for the comparable region at the opposing weld toe, as seen in Fig. 3a.

Stage 4: The CMM surface data are combined, averaged and smoothed into one single 2D contour surface of deformations, Fig. $3 b$.
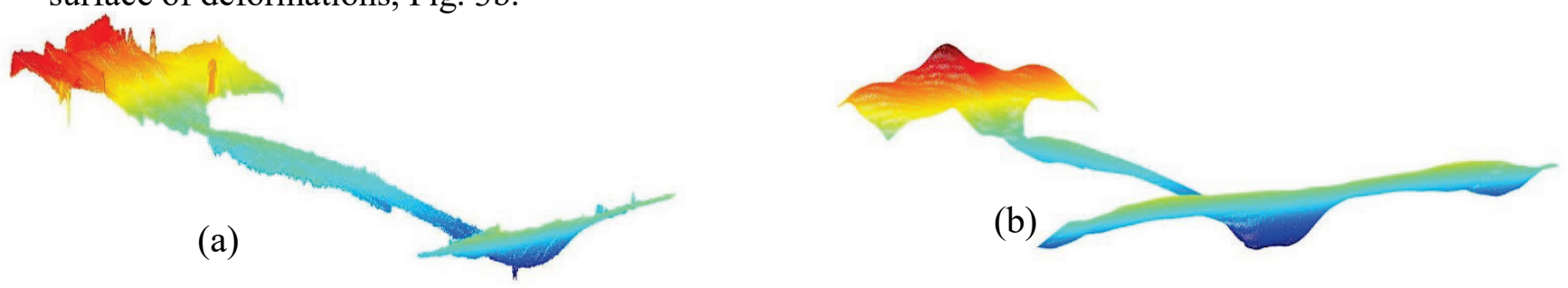

Fig. 3: (a) Raw CMM data (2D and 3D projections), (b) Smoothed CMM data (2D and 3D projections)

The CMM data smoothing was performed for the whole surface after data repairs. Aggressive smoothing leads to loss of details in the residual stress field which can lead to an apparent reduction in the peak magnitudes. Consequently a sensitivity trial with multiple smoothing coefficients was performed to find the minimum level of smoothing that removed excess noise but preserved the details of the residual stress field.

Stage 5: The new contour surface is inverted and introduced into a 3D finite element (FE) model as a boundary condition to reconstruct the original out-of-plane residual stress state of the specimen. Therefore a 3D FE model of the T-section specimen with dimensions limited to the $300 \mathrm{~mm}$ wide region of interest was created. The model contained about 260,000 elements of type C3D8R. Boundary conditions were used to constrain the contour cut surface in the $\mathrm{X}$ and $\mathrm{Y}$ directions in order to have the model acting as a rigid body, Fig. 3a. Then a fine mesh with a node spacing of $0.5 \mathrm{~mm}$ in the weld regions and $1 \mathrm{~mm}$ elsewhere was generated. Finally the displacement data was introduced into the model at the FE nodes corresponding to the matrix elements. The longitudinal residual stresses were then obtained from the FE model after a general static step related to the introduced displacements and the requirement for the stresses across the cut surface to be in equilibrium. A sensitivity study of the mesh and the smoothing of the displacement data (from no smoothing to very aggressive smoothing) was carried out. Only results using optimum mesh and data smoothing are presented.

The ICHD measurement. The ICHD measurements represent the relaxed and redistributed residual stresses present after the contour cut. Therefore to obtain the initial residual stresses at the ICHD measurement locations before the contour cut, the residual stresses obtained from the contour measurements were then taken into account, more specifically the reconstructed in-plane residual stresses from the finite element analysis stage of the contour technique. A superimposition of the measured and reconstructed residual stresses at the measurement locations was therefore carried out for ICHD1, ICHD2 and ICHD3 to obtain the "corrected" residual stresses. The "corrected" residual stresses are meant to be the initial residual stresses at these locations before any material removal. It is important to note that the reconstructed residual stresses from the mirrored analysis of the contour measurement were used for this superimposition since they are thought to be the most accurate.

\section{Results and discussion}

The iDHD, Contour and ICHD measurements were converted into residual stresses using a Young's Modulus, E, and a Poisson's ratio for the parent material. The analyses used for the DHD/iDHD technique assumed isotropic, plane stress conditions; therefore the Poisson's ratio was not required for the iDHD analyses. Each of the measured stress components was normalised with the yield strength $(\sigma y)$ and is plotted as a function of the normalised depth from the plate. 
Fig. $3 \mathrm{~b}$ illustrates the map of the longitudinal residual stresses obtained from the mirrored contour analysis. The symmetric field of displacements generated a balanced residual stress field perfectly symmetric about the stiffener which is very similar to the residual stress field obtained from the original contour measurement for side 1 containing weld toe 1 .
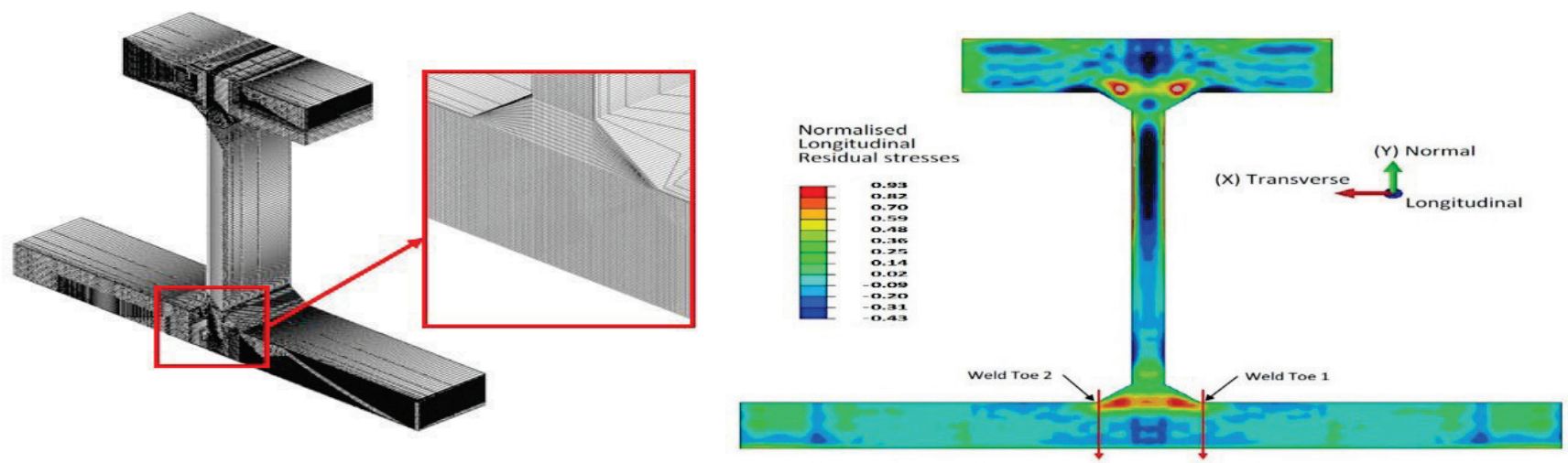

Fig. 3: (a) 3D FE model of the DCNS T-section specimen., (b) 2D Map of the longitudinal residual stresses obtained from the mirrored Contour analysis.

Fig. 4 shows the comparison between the iDHD, the contour and the ICHD measurement in the transverse and longitudinal direction.

The DHD technique measured similar shaped longitudinal and transverse residual stress profiles with the longitudinal and transverse residual stresses being the most tensile throughout. The results showed relatively low tensile residual stresses around $0.15 \sigma_{\mathrm{y}}$ and $0.3 \sigma_{\mathrm{y}}$ a near to the weld toe surface in the transverse and longitudinal directions respectively. The longitudinal and transverse residual stresses both increased to initial peaks of $0.6 \sigma_{\mathrm{y}}$ and $0.35 \sigma_{\mathrm{y}}$ respectively at a depth of $0.07 \mathrm{t}_{0}$. The initial peaks were measured by the iDHD technique having found that the standard DHD technique experienced a small level of plasticity in this region as expected. The longitudinal and transverse residual stresses then decreased slightly before increasing again to maximum tensile peaks of about $0.7 \sigma_{\mathrm{y}}$ and $0.35 \sigma_{\mathrm{y}}$ respectively at a depth of $0.18 \mathrm{t}_{0}$. Finally, the longitudinal and transverse residual stresses decreased into compression at roughly $0.28 \mathrm{t}_{0}$ deep to reach low compressive values of roughly $-0.12 \sigma_{\mathrm{y}}$ and $-0.2 \sigma_{\mathrm{y}}$ respectively thereafter.

The ICHD measurements were carried out on the Contour cut surfaces after completion of the Contour measurement. The ICHD measurements represent the relaxed and redistributed residual stresses present after the contour cut. Therefore to obtain the initial residual stresses at the ICHD measurement locations before the Contour cut, the residual stresses obtained from the Contour measurements were then taken into account, more specifically the reconstructed in-plane residual stresses from the finite element analysis stage of the contour technique. A superimposition of the measured and reconstructed residual stresses at the measurement locations was therefore carried out for ICHD1, ICHD2 and ICHD3 to obtain the "corrected" residual stresses. It is important to note that the reconstructed residual stresses from the mirrored analysis of the contour measurement were used for this superimposition due to being the most accurate. The "corrected" residual stress results for ICHDs 2 and 3 are shown in Fig.4a.

Finally, the results from the mirrored contour analysis are compared with the longitudinal residual stresses obtained from the iDHD measurement at weld toe 1 in Fig.4b. Overall, the mirrored contour measurement did not find the two residual stress peaks at the surface, nor did it find the steep stress gradient measured using DHD. This was probably due to a loss in depth resolution of the Contour results due to too much smoothing of the CMM raw data within the analysis. However, the general trend and peak residual stresses of $0.6 \sigma_{\mathrm{y}}$ and $0.73 \sigma_{\mathrm{y}}$ for iDHD and $0.7 \sigma_{\mathrm{y}}$ for mirrored 
contour were similar. Also both measurement techniques showed residual stresses around $0.3 \sigma y$ near to the weld toe surface and low compression at a depth around $0.4 \mathrm{t}_{0}$.
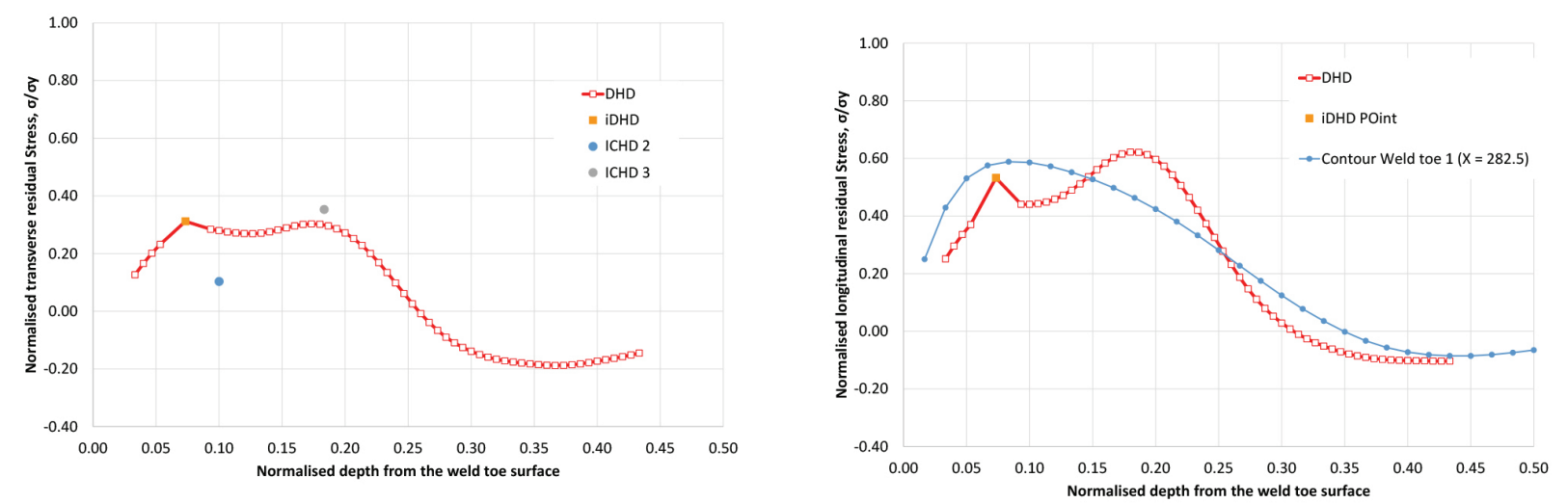

Fig.4: Comparison made between the transeverse (a) and longitudinal (b) residual stresses obtained from the mirrored Contour analysis and the iDHD measurements.

\section{Conclusion}

In the present work, DHD, ICHD and contour method have been coupled to measure the residual stress field within a complex welded part.

DHD and contour methods gave similar results in terms longitudinal stress under the fillet weld. ICDH and DHD results were also relatively close in terms of transverse stress but some scatter between the data was observed. Regarding the contour method, its application to such a complex part revealed all the interest of this method to fully describe the out-of-plane stress field. However, the involved contour cut induced artefacts and thereby scatter in the obtained results. Finally, if the occurrence of plastic stress relaxation is taken into account during DHD with local use of iDHD, the correction of plastic relaxation occurring during the contour method appears as a challenge to tackle.

\section{Acknowledgements}

This work is part of the research programm MUSICAS, which is partly financed by the French Single Interministerial Fund (FUI).

\section{References}

[1] R. H. Leggatt, D. J. Smith, S. Smith, and F. Faure, "Development and experimental validation of the deep hole method for residual stress measurement", Journal of Strain Analysis for Engineering Design, Vol 31, No 3, pp 177-186, (1996). https://doi.org/10.1243/03093247V313177

[2] Kingston, E. K., et al., "Novel applications of the deep-hole drilling technique for measuring through- thickness residual stress distributions", Journal of ASTM International, Vol 3, No 4, (2006). https://doi.org/10.1520/JAI12568

[3] Hosseinzadeh, F. et al. "Towards good practice guidelines for the contour method of residual stress measurement", Journal of Engineering (2014).

[4] Traore, Y. et al. "Measurement of the Residual Stress Tensor in a Compact Tension Weld Specimen”, Experimental Mechanics, 53(4):605-618. https://doi.org/10.1007/s11340-012-9672-7 